Supporting Information for

\title{
Optimization of Surface Loading of the Silatrane Anchoring Group on $\mathrm{TiO}_{2}$
}

\author{
Jennifer L. Troiano, Robert H. Crabtree and Gary W. Brudvig* \\ Department of Chemistry, Yale University, 225 Prospect Street, New Haven, Connecticut \\ 06520-8107, United States \\ and Energy Sciences Institute, Yale University, 520 West Campus Drive, West Haven, \\ Connecticut, 06516, United States
}

*Corresponding author. E-mail address: gary.brudvig@yale.edu 
Materials. All materials were purchased from Aldrich or Alfa-Aesar and used without further purification unless otherwise noted. $p$-Aminophenyltrimethoxysilane, 90\% purity, (3,3,3trifluoropropyl)trimethoxysilane, $98 \%$, and methylsilatrane were purchased from Gelest. TiNanoxide T/SP paste was purchased from solaronix. The particles are anatase and 15-20 nm. Anhydrous acetonitrile was purified with a Solvent Dispensing System. Triethanolamine was dried with Linde type $4 \mathrm{~A}$ molecular sieves for $24 \mathrm{~h}$ at room temperature in the dark. ${ }^{1}$ Millipore water was used in all cases.

Determination of Surface Loading. Surface loading was calculated using UV-Visible

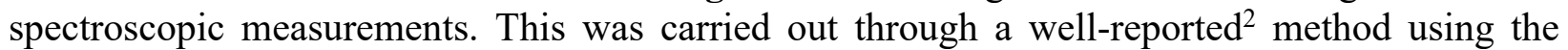
formula,

$$
\Gamma\left(\mathrm{mol} \cdot \mathrm{cm}^{-2}\right)=\frac{A(\lambda)}{1000 \times \varepsilon}
$$

where $A(\lambda)$ is the absorbance at wavelength $\lambda$, and $\varepsilon$ is the molar absorptivity at wavelength $\lambda$. Molecular 1-(phenyldiazenyl)naphthalen-2-ol has a molar absorptivity of $14,500 \mathrm{M}^{-1} \cdot \mathrm{cm}^{-1}$ at $480 \mathrm{~nm} .{ }^{3}$ Since the silatrane anchoring group is not electronically coupled with the surface, we estimated the molar absorptivity of the surface bound compound is similar so we used this number along with the corrected absorbance to calculate surface loading. The corrected absorbance of any thin film was determined by subtracting the measured absorbance of bare $\mathrm{TiO}_{2}$ at $\lambda_{\max }$ from the measured absorbance of the sensitized thin film at this same wavelength. Surface effects and molecular interactions contributed to some deviation of $\lambda_{\max }$, but no more than $\pm 4 \mathrm{~nm}$. 


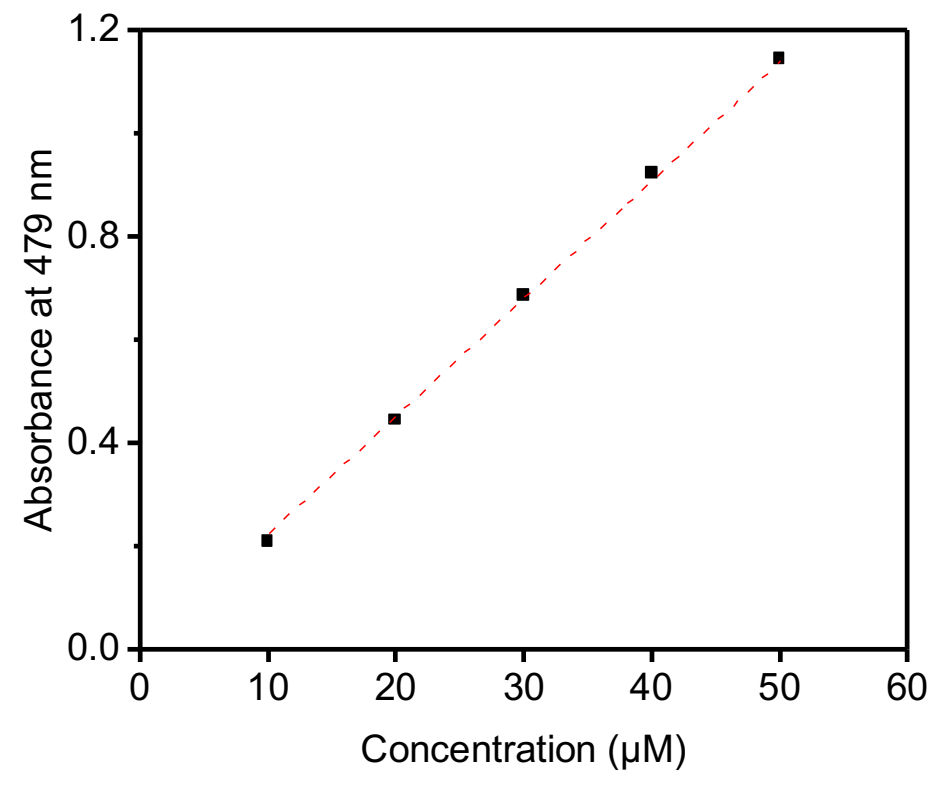

Figure S1. Calibration curve of 2 in $\mathrm{MeCN}$ measured at its $\lambda_{\max }$ of $479 \mathrm{~nm}$. The equation of the linear trendline is $\mathrm{y}=0.02283 \mathrm{x}$ where the slope indicates that the molar absorptivity of the compound is $22,830 \mathrm{M}^{-1} \cdot \mathrm{cm}^{-1}$. 


\section{${ }^{1}$ H NMR Spectroscopy}
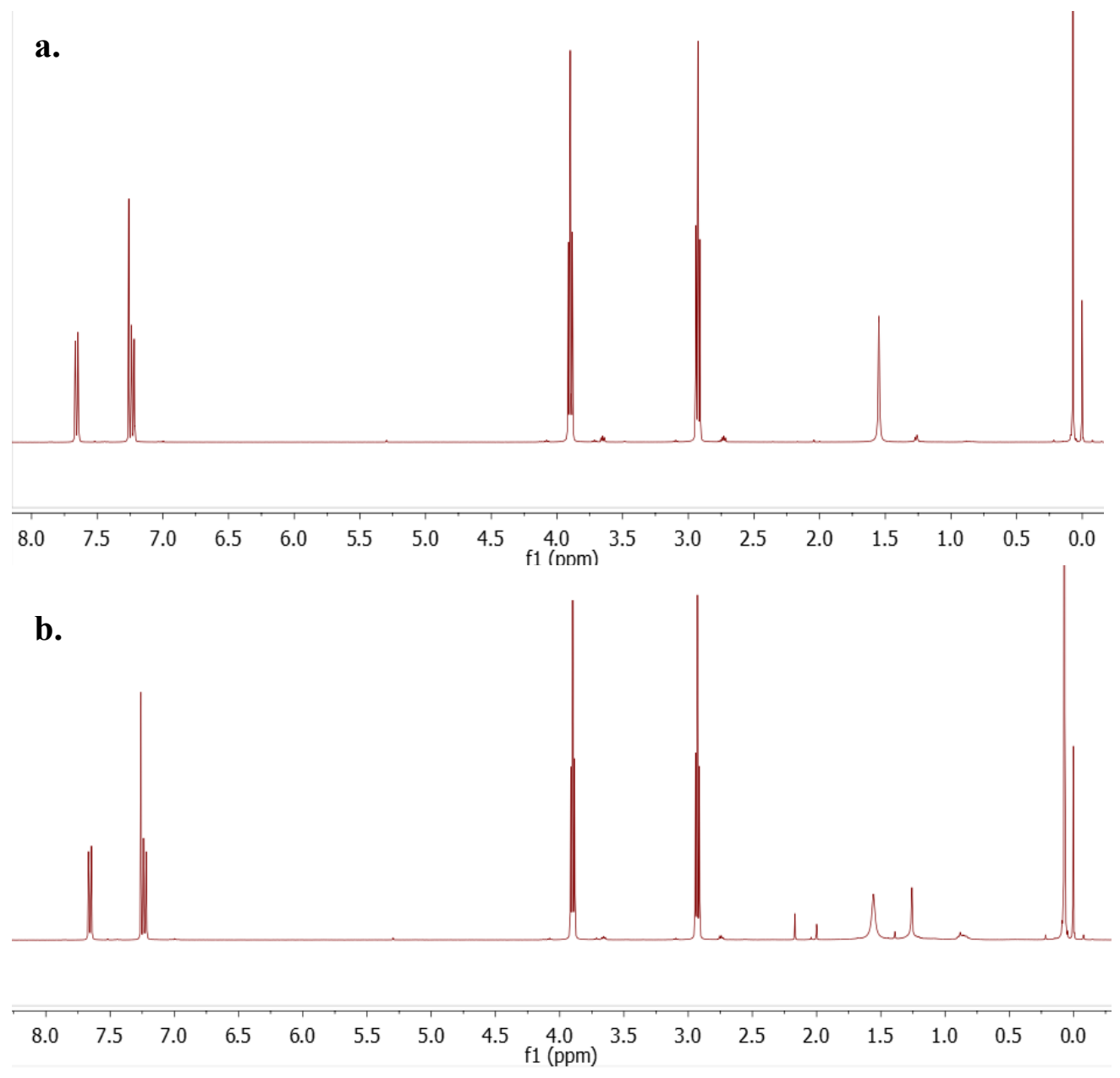

Figure S2. ${ }^{1} \mathrm{H}$ NMR spectrum of 3 in $\mathrm{CDCl}_{3}$ a) before sensitization and b) after sensitization of $\mathrm{TiO}_{2}$ thin film under typical conditions. 


\section{Surface Loading}

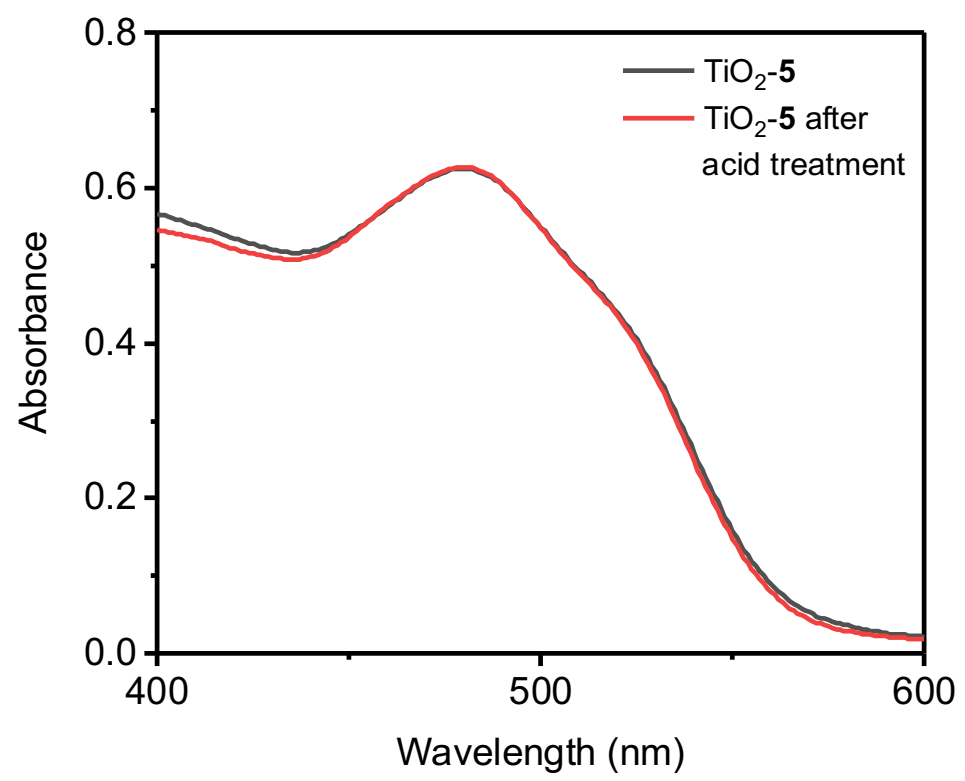

Figure S3. UV-Visible absorption spectra comparing the absorbance of $\mathrm{TiO}_{2}-\mathbf{5}$ before (black) and after (red) acid treatment ${ }^{\mathrm{a}}$.

${ }^{a}$ Acid treatment is described in Experimental Section. Thin films are soaked for $2 \mathrm{~h}$ in $0.1 \mathrm{M} \mathrm{H}_{2} \mathrm{SO}_{4}$ aqueous solution adjusted to $\mathrm{pH} 2$ with $\mathrm{KOH}$.

Table S1. Surface Loading of $\mathbf{5}$ on $\mathrm{SnO}_{2}$ after Loading under Various Conditions ${ }^{\mathrm{a}}$

\begin{tabular}{|l|l|}
\hline Sensitization Conditions & Surface Loading $\left(\mathrm{nmol} \mathrm{cm}^{-2}\right)$ \\
\hline $\mathrm{SnO}_{2} \mathbf{- 3 - 5}$ & $9 \pm 2$ \\
\hline $\mathrm{SnO}_{2} \mathbf{- 3}$-Acid-5 & $18 \pm 2$ \\
\hline $\mathrm{SnO}_{2} \mathbf{- 5}$ & $14 \pm 1$ \\
\hline
\end{tabular}

${ }^{a}$ Comparison of second loading with $\left(\mathrm{SnO}_{2}-3\right.$-acid-5) and without $\left(\mathrm{SnO}_{2}-3-5\right)$ prior TEOA removal to first loading $\left(\mathrm{SnO}_{2}-\mathbf{5}\right)$. Surface loading is calculated as described with eq. S1 using the solidstate absorbance at the appropriate $\lambda_{\max } . \mathrm{n}=2$ for all conditions and the standard deviation is included. 


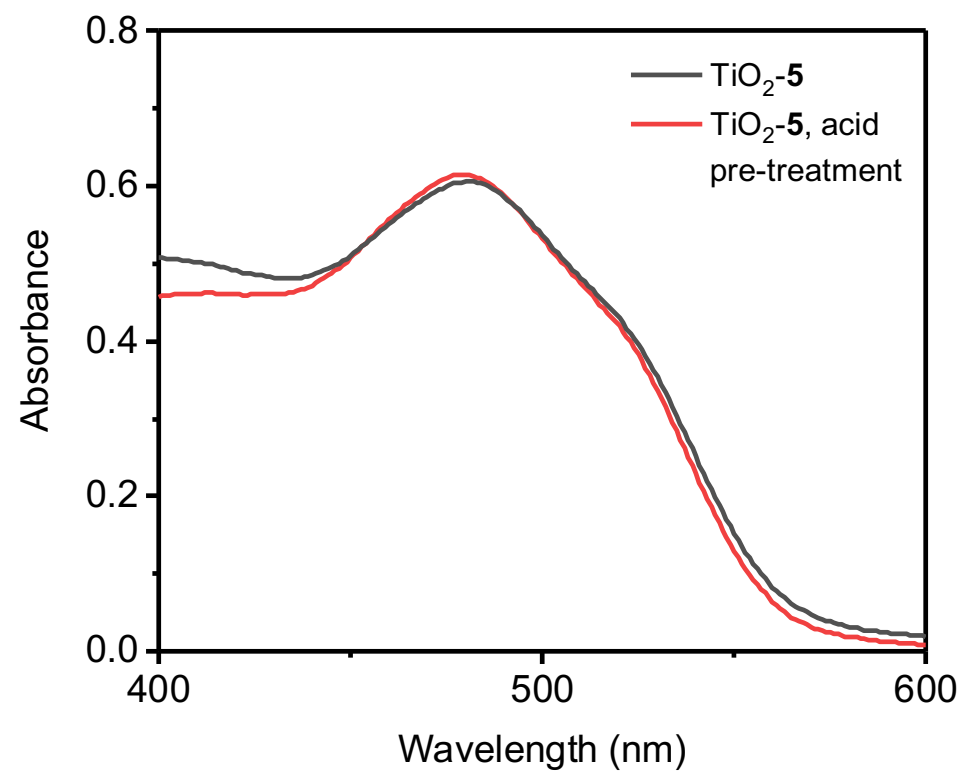

Figure S4. UV-Visible absorption spectra comparing the absorbance of $\mathrm{TiO}_{2}-\mathbf{5}$ when $\mathbf{4}$ was loaded under typical conditions (black) versus when $\mathbf{4}$ was loaded after an acid pre-treatment ${ }^{\mathrm{a}}$ (red).

${ }^{a}$ Acid pre-treatment was the same as the acid treatment used after first sensitization to remove TEOA. Treatment, as noted in experimental section, involves soaking $\mathrm{TiO}_{2}$ thin films in $0.1 \mathrm{M}$ $\mathrm{H}_{2} \mathrm{SO}_{4}$ adjusted to $\mathrm{pH} 2$ with $\mathrm{KOH}$. 


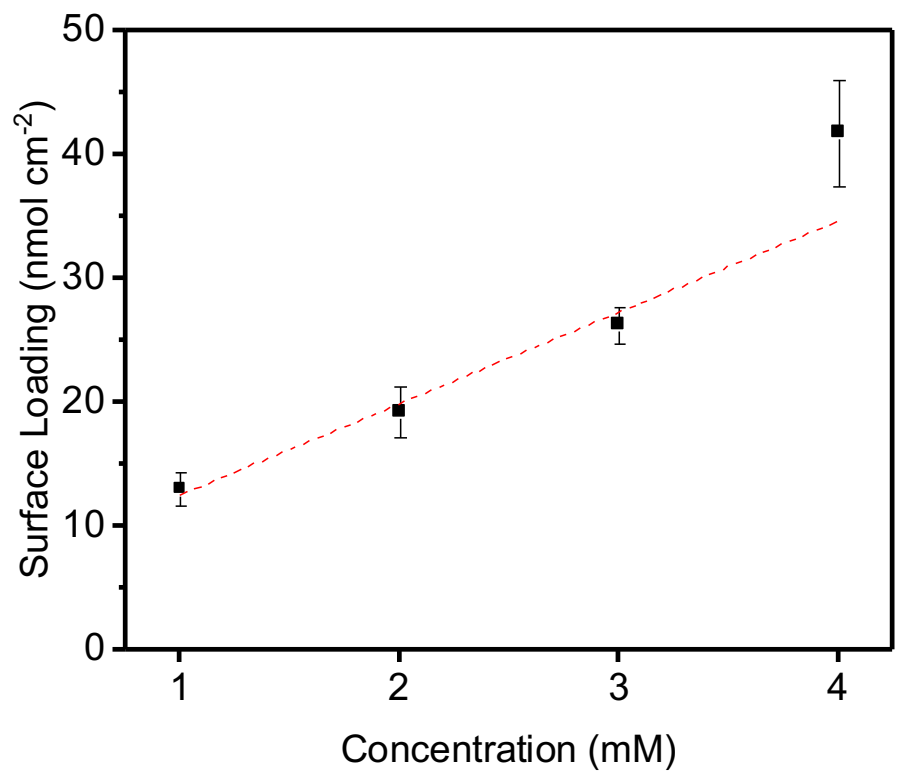

Figure S5. The surface loading of $\mathrm{TiO}_{2}-\mathbf{5}$ versus concentration of the sensitization solution of $\mathbf{4}$ in $\mathrm{MeCN}$ with its linear trendline. The equation of the trend line is $\mathrm{y}=7.37 \mathrm{x}+5.23$ with an $\mathrm{R}^{2}$ value of $0.953 ; \mathrm{n}=2$ for each concentration with error bars showing the standard deviation.

Table S2. Surface Loading of $\mathrm{TiO}_{2}-5$ sensitized with $4 \mathrm{mM} 4$ and various concentrations of benzoic acid additive.

\begin{tabular}{|l|l|} 
Sensitization Conditions & \multicolumn{1}{l|}{ Surface Loading $\left(\mathrm{nmol} \mathrm{cm}^{-2}\right)$} \\
\hline $4 \mathrm{mM} \mathrm{4,} \mathrm{no} \mathrm{benzoic} \mathrm{acid}$ & $42 \pm 4$ \\
\hline $4 \mathrm{mM} \mathrm{4,2} \mathrm{mM}$ benzoic acid & 58 \\
\hline $4 \mathrm{mM} \mathrm{4,50} \mathrm{mM} \mathrm{benzoic} \mathrm{acid}$ & $103 \pm 7$ \\
\hline $4 \mathrm{mM} \mathrm{4,100} \mathrm{mM} \mathrm{benzoic} \mathrm{acid}$ & 51 \\
\hline
\end{tabular}




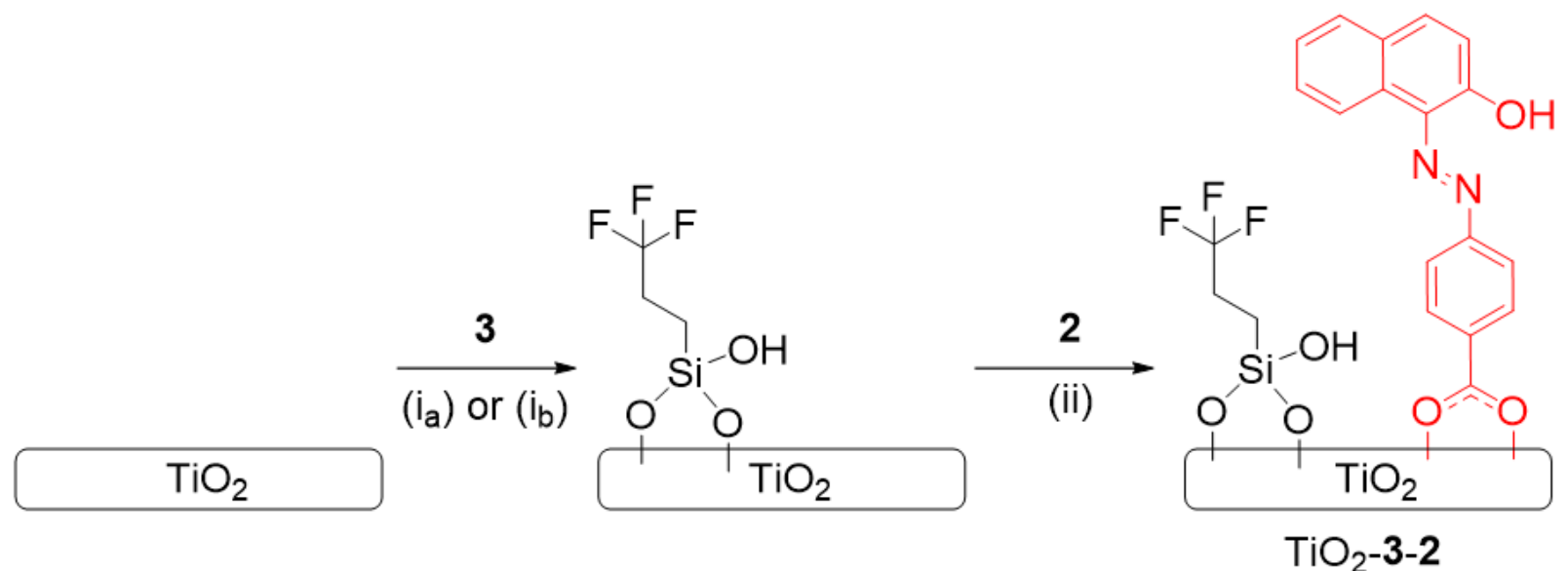

Scheme S1. Process ${ }^{\mathrm{a}}$ for derivatization of $\mathrm{TiO}_{2}$ thin film to compare surface coverage of $\mathbf{3}$ with and without benzoic acid additive.

a $\left(i_{a}\right)$ sensitization with 3 in MeCN ( $i_{b}$ ) sensitization with 3 in MeCN with $50 \mathrm{mM}$ benzoic acid (ii) sensitization with 2 in MeCN. Details in Experimental Section.

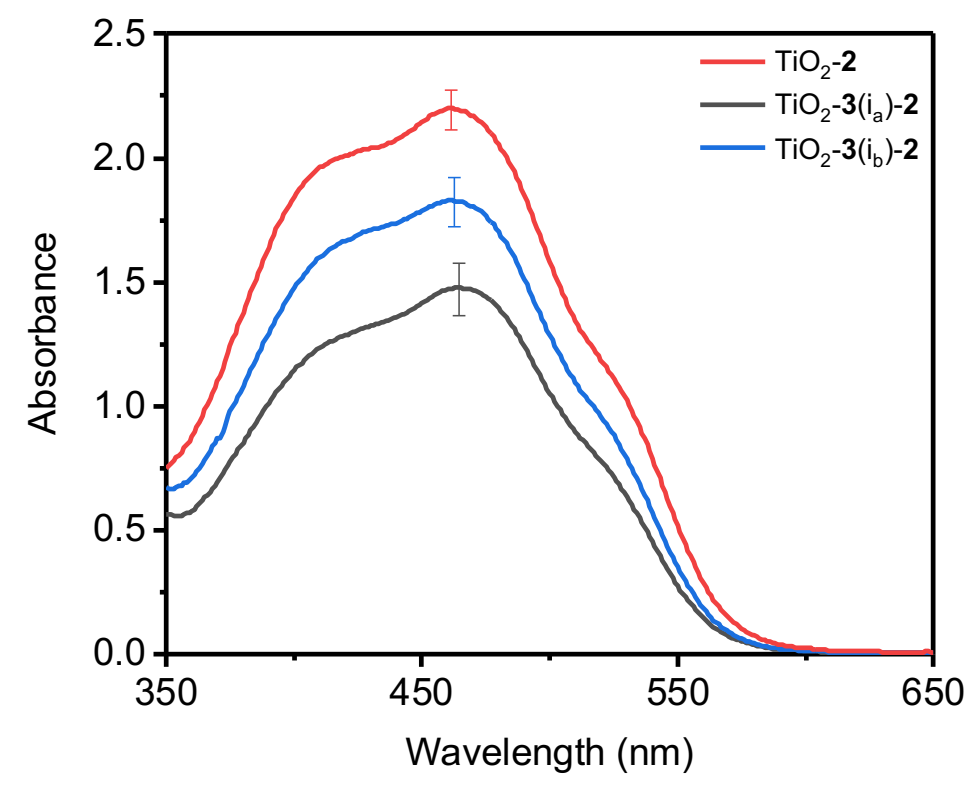

Figure S6. UV-Visible absorption spectra of $\mathrm{TiO}_{2}-2$ (red) compared with that of $\mathrm{TiO}_{2}-\mathbf{3 - 2}$ with ( $\left.i_{a}\right)$ (black) and without ( $i_{b}$ ) (blue) benzoic acid additive for sensitization with 3 . Increased absorbance from $\mathbf{2}$ indicates lower loading of $\mathbf{3}$ on the surface as $\mathbf{2}$ is taken to measure open surface sites. 


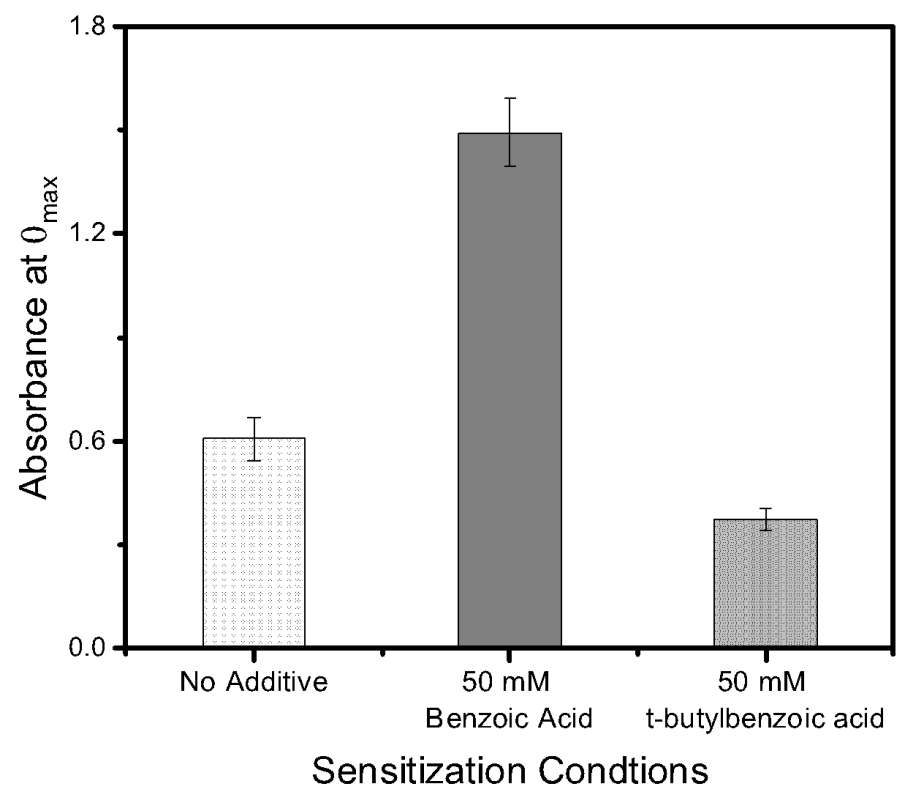

Figure S7. Comparison of absorbance of $\mathrm{TiO}_{2}-\mathbf{5}$ formed through sensitization with $4 \mathrm{mM} 4$ in $\mathrm{MeCN}$ containing no additive, $50 \mathrm{mM}$ benzoic acid, and $50 \mathrm{mM}$ t-butylbenzoic acid.

\section{References:}

(1) W. Armarego and C. Chai, in Purification of Organic Chemicals, Elsevier, Oxford, UK, the $6^{\text {th }}$ edition, 2009, Ch. 4, p.184.

(2) Trammell, S. A.; Meyer, T. J. Diffusional Mediation of Surface Electron Transfer on $\mathrm{TiO}_{2}$. J. Phys. Chem. B 1999, 103 (1), 104-107.

(3) Taniguchi, M.; Lindsey, J. S. Database of Absorption and Fluorescence Spectra of $>300$ Common Compounds for Use in PhotochemCAD. Photochem. Photobiol. 2018, 94 (2), 290-327. 